\title{
Psychological characteristics and fatal ischaemic heart disease
}

\author{
A Haines, J Cooper, T W Meade
}

\begin{abstract}
Objective-To determine the relation between psychological characteristics and subsequent fatal ischaemic heart disease (IHD) events.

Design-Prospective study of participants in the Northwick Park heart study (NPHS) recruited between 1972 and 1978 and followed up for fatal events until 1997.

Setting-Three occupational groups in north west London.

Subjects-1408 white men without a history of myocardial infarction aged 40-64 years at entry who completed a Crown-Crisp experiential index form (CCEI).

Main outcome measure-Fatal IHD during follow up.

Results-A one point increase in the score on the obsessionality/obsessional neurosis subscale was associated with a relative risk of fatal IHD of 1.08 (95\% confidence interval (CI) 1.02 to 1.15). For the functional somatic complaint subscale the relative risk was also 1.08 (95\% CI 1.02 to 1.15$)$. In the case of the total score the relative risk of fatal IHD was 1.28 (95\% CI 1.09 to 1.50) for a 10 point increase. The associations were independent of age, social class, and known cardiovascular risk factors. In the case of phobic anxiety, which had previously been found to be associated with fatal IHD in NPHS, the association was evident in the first 10 years of follow up but overall the relative risk was only 1.07 (95\% CI 0.99 to 1.15$)$ for a one point increase in the score.

Conclusion-Scores on two of the subscales of the CCEI and the total score are significantly associated with fatal IHD on long term follow up independently of other known risk factors. (Heart 2001;85:385-389)
\end{abstract}

Keywords: cohort study; ischaemic heart disease; psychological factors

There has been extensive interest in the possible role of psychosocial factors in the causation of ischaemic heart disease (IHD) events. We have previously described an association between phobic anxiety and fatal $\mathrm{IHD}^{1}$ using the Crown-Crisp experiential index (CCEI) form and this has been confirmed in a cohort of over 33000 American male health professionals. ${ }^{2}$ In an earlier study ${ }^{3}$ on a small sample of men using the CCEI, those who had suffered a myocardial infarction during the follow up period had higher scores at entry than those who had not on the free floating anxiety and functional somatic complaint subscales. In addition the investigators selected individual questions that best discriminated between those who did and those who did not subsequently experience a myocardial infarction, but this approach may lead to spurious association by chance. ${ }^{4}$

A recent review of negative emotions in the causation of IHD concluded that, of anxiety, anger, and depression, the evidence was strongest in the case of anxiety. ${ }^{5}$ A systematic review of psychosocial factors in the aetiology and prognosis of IHD also showed that anxiety was consistently related to events in three prospective cohort studies. ${ }^{6}$

Other investigations have reported the psychological characteristics of patients who have developed symptoms of IHD, in which case the characteristics may result from clinical events rather than antedating them. Few studies, particularly in the UK, report long term follow up of the association between psychosocial charac- teristics and IHD. This paper reports the follow up of white men in the Northwick Park heart study from 1972 to 1997 for fatal events and substantially extends the period of observation described in our earlier paper.

\section{Methods}

The main purpose of the Northwick Park heart study was to investigate the thrombotic component of IHD by including measures of haemostatic function along with more familiar risk factors. Its design, conduct, and principal results have been described elsewhere together with the laboratory methods and details of the clinical examination. ${ }^{78}$ Participants were recruited from 1972 to 1978 by sending invitation letters to employees in three occupational groups (a food processing company, the post office, and local government workers) in north west London. The initial response to an invitation to participate was about $80 \%$. The response rate is a slight approximation because men were moving from one department to another and from one shift to another during the course of the study in the largest of the three occupational groups (the food processing workers), which made it difficult to ascertain the true denominator precisely. This report is restricted to white males aged 40-64 years at recruitment (as in our earlier paper ${ }^{1}$ ) because of small numbers in other groups. All participants were given a CCEI form at the recruitment examination and asked to return it in a prepaid envelope after completion. This index is a standardised self rating inventory of 
Table 1 Correlations between subscales on the Crown-Crisp experiential index

\begin{tabular}{lllllll}
\hline & Anxiety & Phobia & Obsession & Somatic & Depression & Hysteria \\
\hline Anxiety & 1 & & & & \\
Phobia & $r=0.39$ & 1 & & & \\
& $\mathrm{p}<0.0001$ & & & & \\
Obsession & $r=0.43$ & $r=0.29$ & 1 & & & \\
& $\mathrm{p}<0.0001$ & $\mathrm{p}<0.0001$ & & & & \\
Somatic & $r=0.45$ & $r=0.28$ & $r=0.30$ & 1 & & \\
& $\mathrm{p}<0.0001$ & $\mathrm{p}<0.0001$ & $\mathrm{p}<0.0001$ & & \\
Depression & $r=0.50$ & $r=0.34$ & $r=0.32$ & $r=0.40$ & 1 & \\
& $\mathrm{p}<0.0001$ & $\mathrm{p}<0.0001$ & $\mathrm{p}<0.0001$ & $\mathrm{p}<0.0001$ & \\
Hysteria & $r=0.25$ & $r=0.16$ & $r=0.17$ & $r=0.16$ & $r=0.24$ & 1 \\
& $\mathrm{p}<0.0001$ & $\mathrm{p}<0.0001$ & $\mathrm{p}<0.0001$ & $\mathrm{p}<0.0001$ & $\mathrm{p}<0.0001$ & \\
\hline
\end{tabular}

48 questions allowing scores of $0-16$ on each of six scales that measure free floating anxiety, phobic anxiety, obsessionality/obsessional neurosis, functional somatic complaint, depression, and "hysteria" (extraversion). It has been validated in psychiatric outpatients and used extensively in other studies..$^{9-11}$

Of the 1511 white men aged 40-64, 1457 completed the CCEI form. Follow up to establish mortality was through automatic notification of death through the National Health Service central register from April 1972 to July 1997. Forty nine men with evidence of a previous myocardial infarction at entry to the study have been excluded from the analyses presented here. These men had previously been shown to have higher scores on the free floating anxiety and functional somatic complaint scales. ${ }^{1}$ The analyses presented here are therefore based on data from 1408 men. The mean and range of the time from recruitment to death or the end of the follow up period was 20.9 years ( 7 days to 27.7 years).

When notifications of death were received further details of the patient's medical history, the results of diagnostic tests, including ECGs and cardiac enzymes, and the results of postmortem examinations where available were sought from general practitioners, hospitals, and coroners. Information obtained in this manner was then assessed by three doctors independently of the study who decided whether the deaths were attributable to IHD according to the World Health Organization criteria. ${ }^{12}$ During the total follow up period 127 men were considered to have had fatal IHD. Non-fatal events are not included in this report because $\mathrm{we}^{1}$ and others ${ }^{2}$ previously demonstrated that the association of scores on the CCEI is predominantly with fatal rather than non-fatal events. In any case information on non-fatal events was available only until 1984 and was reported in our previous paper.

\section{STATISTICAL METHODS}

Spearman rank correlation after adjustment for age and smoking was used to test intercorrelation between scores on the subscales of the CCEI and correlations between the scores on the subscales and other cardiovascular risk factors. Differences of scores on the CCEI subscales between men of different social classes were tested using the Kruskal Wallis test. Scores on most of the subscales of the index have previously been shown to be related to age and smoking habits. ${ }^{13}$ These variables together with social class, body mass index (BMI), systolic blood pressure, factor VII activity, serum cholesterol, and plasma fibrinogen were included in survival analyses using Cox's proportional hazards model, ${ }^{14}$ which takes into account the varying lengths of follow up. The data are presented with men dying of causes other than IHD included in the survival analyses and censored at the time of the death. The sensitivity of the findings to including and removing the data from men dying of other causes was assessed.

\section{Results}

ASSOCIATIONS BETWEEN SCORES ON THE CCEI SUBSCALES AND OTHER VARIABLES

There was a weak positive association between both BMI $(r=0.06, \mathrm{p}=0.04)$ and plasma fibrinogen $(r=0.06, \mathrm{p}=0.02)$ and the score on the functional somatic complaint scale.

Scores on the phobic anxiety $(\mathrm{p}=0.0001)$, obsessionality/obsessional neurosis $(p=0.001)$, functional somatic complaint $(\mathrm{p}=0.0001)$, and depression $(p=0.0001)$ subscales and the total score $(p<0.0001)$ were significantly higher in men of lower than in those of higher social class, comparing scores for social classes I, II, and III non-manual with those for III manual, IV, and V. However, a change in the score on the obsessionality/obsessional neurosis subscale of 1 (the difference in score between social class I, median score 6 and social class V, median score 7) is associated with a relative risk of IHD of only 1.08 (95\% confidence interval (CI) 1.02 to 1.15).

\section{INTERCORRELATIONS BETWEEN SCORES ON THE} SUBSCALES

Correlations between subscales of the index ranged from $r=0.16(p<0.0001)$ for the association between functional somatic complaint and hysteria to $r=0.50(p<0.0001)$ for the association between free floating anxiety and depression (table 1).

ASSOCIATIONS OF SCORES ON THE CCEI WITH FATAL IHD AND DEATHS FROM OTHER CAUSES The mean scores on the functional somatic complaint and obsessionality/obsessional neurosis subscales of the CCEI were significantly higher at entry in men who subsequently died of IHD after adjustment for all the risk factors listed above (table 2). The total score was also significantly higher among those who developed fatal IHD than among survivors after adjustment for all the above risk factors. The changes in relative risk per unit change in score on the subscales and per 10 unit change in the total score are also shown in table 2 . The sensitivity of the results to the exclusion of men dying of non-cardiovascular causes from the survival analysis was tested by reanalysing the data with their data excluded, but this did not alter the results appreciably.

The relative risks by approximate quintiles of scores on the three subscales shown to be associated with fatal IHD and the total score are shown in table 3 . These were adjusted for other cardiovascular risk factors listed above in the Methods section, and the $\mathrm{p}$ values for trend 
Table 2 Comparison of adjusted scores on the subscales of the CCEI and the total score for surviving men and those who died of IHD

\begin{tabular}{|c|c|c|c|c|c|c|c|}
\hline & Anxiety & Phobic anxiety & $\begin{array}{l}\text { Obsessional } \\
\text { neurosis }\end{array}$ & $\begin{array}{l}\text { Functional somatic } \\
\text { complaint }\end{array}$ & Depression & Hysteria & Total score \\
\hline & \multicolumn{7}{|c|}{ Adjusted scores in survivors $(n=1092)$} \\
\hline Median & 2.5 & 2.4 & 6.2 & 2.7 & 1.9 & 2.8 & 19.3 \\
\hline LQ & 1.1 & 1.0 & 4.0 & 1.2 & 0.6 & 1.1 & 13.4 \\
\hline \multirow[t]{2}{*}{ UQ } & 4.9 & 4.0 & 8.5 & 4.6 & 3.7 & 5.0 & 27.3 \\
\hline & \multicolumn{7}{|c|}{ Adjusted scores in men who developed fatal ischaemic heart disease $(n=127)$} \\
\hline Median & 2.6 & 2.8 & 6.9 & 3.6 & 2.6 & 3.1 & 22.9 \\
\hline LQ & 1.1 & 1.0 & 4.7 & 1.5 & 0.9 & 1.2 & 15.0 \\
\hline UQ & 5.8 & 4.3 & 8.9 & 5.5 & 4.5 & 5.8 & 31.4 \\
\hline Relative risk ${ }^{\star}$ & 1.04 & 1.07 & 1.08 & 1.08 & 1.07 & 1.05 & 1.28 \\
\hline $95 \% \mathrm{CI}$ & 0.99 to 1.10 & 0.99 to 1.15 & 1.02 to 1.15 & 1.02 to 1.15 & 0.99 to 1.15 & 0.99 to 1.12 & 1.09 to 1.50 \\
\hline $\mathrm{p}$ Value & 0.17 & 0.10 & 0.009 & 0.009 & 0.08 & 0.13 & 0.003 \\
\hline
\end{tabular}

^From Cox model for fatal IHD adjusted for age, smoking, BMI, fibrinogen, factor VIIc, systolic blood pressure, cholesterol, and social class. Relative risk is for a one point increase in each subscale and a 10 point increase in the total score.

LQ, lower quartile; UQ, upper quartile

were obtained from Cox's proportional hazards model. For scores on the obsessionality/ obsessional neurosis subscale and the total score there is a generally consistent and significant pattern of increasing relative risk of fatal IHD with increasing score. In the case of the functional somatic complaint scale the trend is significant $(p<0.01)$ when adjusted for age and smoking only but not when adjusted for all risk factors (table 3 ) because of the weak positive associations with BMI, plasma fibrinogen, and social class described above.

For phobic anxiety the relation between the scales and IHD is seen only in those who developed an event less than 10 years after entry in to the study. The association of the score on the phobic anxiety subscale with subsequent fatal IHD is not significant $(p=0.10)$ over the full follow up period. However, there is a linear trend $(p=0.02)$ for the association of phobic anxiety with fatal IHD to diminish with increasing length of follow up (fig 1). There was no significant trend of the strength of the association over time with fatal IHD for the score on the obsessionality/obsessional neuro-

Table 3 Adjusted relative risks (95\% CI) of fatal IHD by approximate quintiles of scores on three of the subscales and the total score

\begin{tabular}{|c|c|c|c|}
\hline Score & $\begin{array}{l}\text { Number of } \\
\text { events }\end{array}$ & Survivors & $R R(95 \% C I)$ \\
\hline \multicolumn{4}{|c|}{ Obsessional neurosis } \\
\hline $0-3$ & 10 & 181 & 1.00 \\
\hline $4-5$ & 17 & 222 & $1.19(0.53$ to 2.64$)$ \\
\hline $6-7$ & 33 & 265 & $1.69(0.81$ to 3.49$)$ \\
\hline $8-9$ & 38 & 222 & $2.64(1.29$ to 5.43$)$ \\
\hline $10+$ & 29 & 202 & $1.88(0.90$ to 3.97$)$ \\
\hline $\mathrm{p}$ (linear trend) & & & 0.01 \\
\hline \multicolumn{4}{|c|}{ Functional somatic complaint } \\
\hline $0-1$ & 14 & 222 & 1.00 \\
\hline 2 & 19 & 172 & $1.31(0.62$ to 2.76$)$ \\
\hline 3 & 15 & 168 & $1.30(0.60$ to 2.83$)$ \\
\hline $4-5$ & 32 & 278 & $1.45(0.73$ to 2.85$)$ \\
\hline $6+$ & 47 & 252 & $1.76(0.91$ to 3.40$)$ \\
\hline $\mathrm{p}$ (linear trend) & & & 0.08 \\
\hline \multicolumn{4}{|l|}{ Phobic anxiety } \\
\hline $0-1$ & 32 & 299 & 1.0 \\
\hline 2 & 16 & 207 & $0.74(0.39$ to 1.40$)$ \\
\hline 3 & 18 & 174 & $1.14(0.62$ to 2.12$)$ \\
\hline $4-5$ & 40 & 251 & $1.25(0.76$ to 2.05$)$ \\
\hline $6+$ & 21 & 161 & $1.29(0.71$ to 2.34$)$ \\
\hline $\mathrm{p}$ (linear trend) & & & 0.16 \\
\hline \multicolumn{4}{|l|}{ Total score } \\
\hline $0-14$ & 18 & 241 & 1.0 \\
\hline $15-19$ & 23 & 231 & $1.29(0.67$ to 2.50$)$ \\
\hline $20-25$ & 24 & 218 & $1.23(0.64$ to 2.35$)$ \\
\hline $26-32$ & 23 & 213 & $1.31(0.68$ to 2.50$)$ \\
\hline $33+$ & 39 & 189 & $2.30(1.28$ to 4.13$)$ \\
\hline $\mathrm{p}$ (linear trend) & & & 0.01 \\
\hline
\end{tabular}

Adjusted for age, smoking, BMI, fibrinogen, factor VIIc, systolic blood pressure, cholesterol, and social class sis subscale or the functional somatic complaint subscale (figs 2 and 3 ).

Because of the strong intercorrelations between scores on many of the subscales, when scores on each subscale were adjusted for all other subscales in the statistical analysis, the overall associations of scores on the subscales

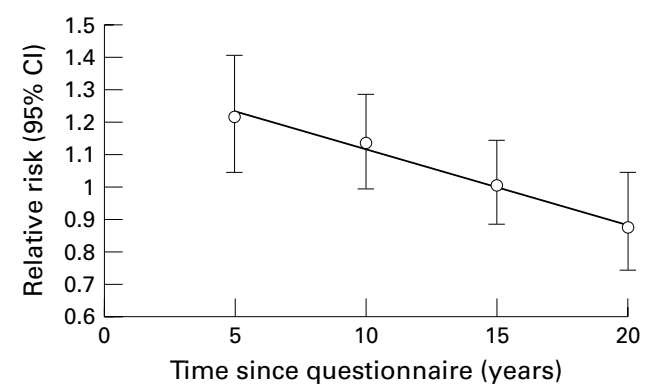

Figure 1 Trend of the relative risk (with 95\% CI) of fatal ischaemic heart disease for a one point change of the score on the phobic anxiety subscale over the period of follow $u p(p=0.02)$.



Figure 2 Trend of relative risk (with 95\% CI) of fatal ischaemic heart disease for a one point change of the score on the obsessionality/obsessional neurosis subscale over the period of follow up.

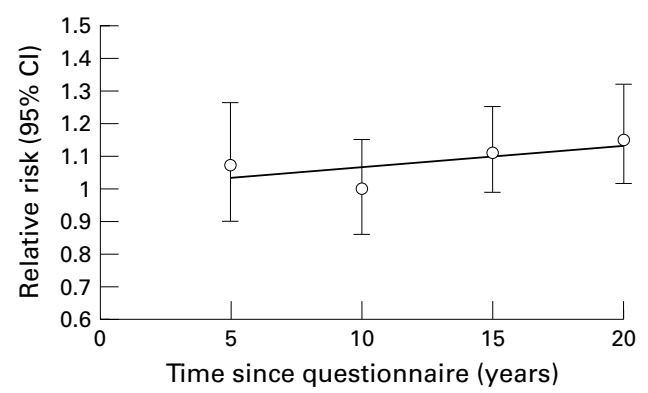

Figure 3 Trend of relative risk (with 95\% CI) of fatal ischaemic heart disease for a one point change of score on the functional somatic complaint subscale over the period of follow up. 
Table 4 Attributable risks of fatal IHD for scores above the median for each variable

\begin{tabular}{lll}
\hline & Percentage & $95 \%$ CI \\
\hline Serum cholesterol & 15.3 & -4.2 to 33.6 \\
Systolic blood pressure & 28.8 & 8.7 to 46.8 \\
Phobic anxiety & 12.7 & -5.8 to 30.6 \\
Obsessionality/obsessional neurosis & 20.3 & 2.0 to 37.3 \\
Functional somatic symptoms & 19.0 & 0.5 to 36.1 \\
Total score & 16.7 & -1.5 to 34.2 \\
\hline
\end{tabular}

Percentages add up to more than $100 \%$ because of intercorrelations between variables.

with fatal IHD were weakened. Nevertheless, scores on both the obsessionality/obsessional neurosis and functional somatic complaint subscales remained significantly associated with fatal IHD. In both cases the relative risk for a one point increase in score was 1.07 (95\% CI 1.00 to $1.15, \mathrm{p}<0.05$ for both variables) when adjusted for age, smoking, all the risk factors, and all other subscales.

There were no significant associations with scores on any of the subscales and subsequent death from cancer. The score on the functional somatic complaint scale for those $(n=57)$ who died from other causes besides cancer and cardiovascular disease was 4.5 (interquartile range 2.4-7.1) compared with those alive at the end of follow up $(n=1092)$, for whom the median score equalled 3.3 (interquartile range $1.6-5.3$ ), $\mathrm{p}=0.01$ (adjusted for age and smoking).

ATTRIBUTABLE RISKS OF SCORES ON THE CCEI FOR FATAL IHD COMPARED WITH "STANDARD" CARDIOVASCULAR RISK FACTORS

To compare the relative contributions of scores on the CCEI to fatal IHD with known cardiovascular risk factors, attributable risks of values above the median were calculated for serum cholesterol, systolic blood pressure, and scores on the functional somatic complaint and obsessionality/obsessional neurosis subscales, as well as the total score. The attributable risks (expressed as a percentage with $95 \%$ CIs) for each variable taking into account other known cardiovascular risk factors as listed in the Methods section are shown in table 4.

This analysis shows that CCEI scores above the median are associated with a broadly similar attributable risk for fatal IHD as values above the median of systolic blood pressure and plasma cholesterol, although the confidence intervals for all estimates are wide.

\section{Discussion}

Scores on two of the subscales and the total score on the CCEI were significantly higher in those who developed fatal IHD over long term follow up than in survivors. These associations were largely independent of other known risk factors despite the associations between scores on the subscales and cigarette smoking, ${ }^{13}$ social class, and, in the case of functional somatic complaint, weak associations with BMI and plasma fibrinogen.

The association of fatal IHD with obsessionality/obsessional neurosis was also noted in our earlier paper but appeared to be weaker than the association with phobic anxiety at that time. However, long term follow up indicates that the association remains consistent over time and, no doubt because of the larger number of fatal events with long term follow up, the association is significant on long term (average 20.9 years) follow up. It seems unlikely that the association is caused by increased detection of disease as a result of a greater tendency for such people to present their symptoms to doctors, because the association is present for fatal IHD.

The association with scores on the functional somatic complaint scale may result from symptoms of physical disease because some of the questions-for example, relating to palpitations and shortness of breath - are likely to be answered positively by those with cardiovascular and perhaps other diseases. However, persistence of the association beyond five years of follow up in participants without any evidence of IHD at entry into the study suggests that it may not be caused by pre-existing physical disease and may reflect somatic symptoms of anxiety. Only scores on the functional somatic complaint scale were associated with other deaths (unrelated to IHD or cancer).

In our previous paper we documented an association between fatal IHD and phobic anxiety score. At that time there did not appear to be any weakening of the relation over time, but the numbers of those followed up for more than five years were limited. In this paper we demonstrate that there is a linear trend of decreasing association between phobic anxiety and fatal IHD with time elapsed from completing the questionnaire. This may be because there are considerable variations in phobic anxiety over time whereas the obsessionality/ obsessional neurosis subscale includes both questions relating to personality traits, which are likely to be stable, and questions related to symptoms that may be more variable. ${ }^{15}$

The mechanism of the associations described is still open to debate. It has been suggested that anxiety may increase the risk of IHD by influencing behaviours, promoting atherogenesis or triggering fatal coronary events. Our results are most compatible with the third of these categories. ${ }^{16}$

Heart rate variability provides a non-invasive measure of cardiac autonomic tone, and reduced variability may be a risk factor for sudden death. A number of reports have linked anxiety and panic disorder to reduced variability of heart rate, ${ }^{16}$ including in men with high levels of phobic anxiety, ${ }^{17}$ which in our study was not found to be associated with fatal IHD on long term follow up. Psychological factors may also affect coronary artery tone. For example, studies have demonstrated vasoconstriction of atherosclerotic segments of coronary arteries in response to recall of an event that had produced anger, ${ }^{18}$ and silent ventricular dysfunction in patients with chronic stable coronary artery disease in response to mental stress in those who tended to respond with anger, aggression or hostility. ${ }^{19}$ Further work is required to determine whether men exhibiting obsessional traits and symptoms or somatic symptoms of anxiety react to mental 
stress in a similar fashion. It is possible that men with high levels of obsessionality or somatic symptoms of anxiety react to the pain and stress of a coronary event in ways that increase their risk of dying.

In view of the therapeutic use of cognitive behavioural therapy for patients with obsessional neurosis, phobias, and other psychological disorders to reduce their symptoms, ${ }^{20}$ there is a case for evaluating the impact of such interventions on the mortality from IHD among men who score highly on the relevant subscales of the CCEI.

We thank Professors Andrew Steptoe and Michael King for helpful comments on this paper, and Professor Ichiro Kawach for useful advice.

1 Haines AP, Imeson JD, Meade TW. Phobic anxiety and ischaemic heart disease. BMF 1987;295:297-9.

2 Kawachi I, Colditz GA, Ascherio A, et al. Prospective study of phobic anxiety and risk of coronary heart disease in men. Circulation 1994;89:1992-7.

3 Crisp AH, Queenan M, D'Souza MF. Myocardial infarction and the emotional climate. Lancet 1984;i:616-9.

4 Thompson SE. Predicting myocardial infarction. Lancet 1984;ii:1021-2.

5 Kubzansky LD, Kawachi I . Going to the heart of the matter: do negative emotions cause coronary heart disease? f Psychosom Res 2000;48:323-37.

6 Hemingway H, Marmot M. Psychosocial factors in the aetiology and prognosis of coronary heart disease: systematic review of prospective cohort studies. BMF 1999;318:1460-7.
7 Meade TW, North WRS, Chakrabarti R, et al. Haemostatic Meade TW, North WRS, Chakrabarti R, et al. Haemostatic function and cardiovascular death:

prospective study. Lancet 1980;i:1050-4

Meade TW, North WRS, in collaboration with Chakrabarti R, Haines A, Stirling Y. Population based distribution of haemostatic variables. Br Med Bull 1977;33:283-6.

9 Crown S, Crisp AH. A short clinical diagnostic self-rating scale for pyschoneurotic patients. The Middlesex Hospital questionnaire. Br f Psychiatry 1966;112:917-23.

10 Crisp AH, Priest RG. Psychoneurotic status during the year following bereavement. F Psychosom Res 1972;16:351-5.

11 Crisp AH, McGuiness B. Jolly fat: the relation between obesity and psychoneurosis in the general population. $B M \mathcal{f}$ 1976;272:7-9.

12 World Health Organization Regional Office for Europe. Myocardial infarction community registers. Public Health in Europe No. 5. Copenhagen: WHO, 1976.

13 Haines AP, Imeson JD, Meade TW. Psychoneurotic profiles of smokers and non-smokers. BMF 1980;280:1422.

14 Hopkins A. P21: regression with incomplete survival data. Hopkins A. P21: regression with incomplete survival data.
In: Dixon WJ, Brown MB, eds. BMDP statistical software. In: Dixon WJ, Brown MB, eds. BMDP statistical software.
Los Angeles: University of California Press, 1983:330-44

5 Crown S, Crisp AH. Manual of the Crown-Crisp experiential Crown S, Crisp AH. Manual of the Crown-Crisp
index. London: Hodder and Stoughton, 1979.

16 Kubzansky LD, Kawachi I, Weiss ST, et al. Anxiety and coronary heart disease: a synthesis of epidemiological, psychological and experimental evidence. Ann Behav Med 1998;20:47-58.

17 Kawachi I, Sparrow D, Vokonas PS, et al. Decreased heart rate variability in men with phobic anxiety. Am $\mathcal{F}$ Cardiol 1995;75:882-5.

18 Boltwood MD, Barr Taylor C, Boutte Burke M, et al. Anger report predicts coronary artery vasomotor response to mental stress in artherosclerotic segments. Am $\mathcal{F}$ Cardiol 1993;72:1361-5.

19 Bury MM, Jain D, Soufier R, et al. Role of behavioural and psychological factors in mental stress induced left ventricular dysfunction in coronary artery disease. $\mathcal{f} \mathrm{Am}$ Coll Cardiol 1993;22:440-8.

20 Enright S. Cognitive behaviour therapy-clinical application. BMF 1997;324:1811-6.

\section{IMAGES IN CARDIOLOGY}

\section{T wave alternans: an electrocardiographic sign of cardiac instability}

A 46 year old African American woman with a history of hypertension and seizure disorder was hospitalised for seizures and aspiration pneumonia. On the second hospital day she suddenly became aphasic with loss of power in her left upper and lower extremities. Her ECG revealed sinus rhythm, prolongation of the QT interval, and beat to beat variation in the $T$ wave amplitude consistent with $\mathrm{T}$ wave alternans (below). Computed tomography scan of the head showed a large right hemispheric stroke with mass effect, following which she
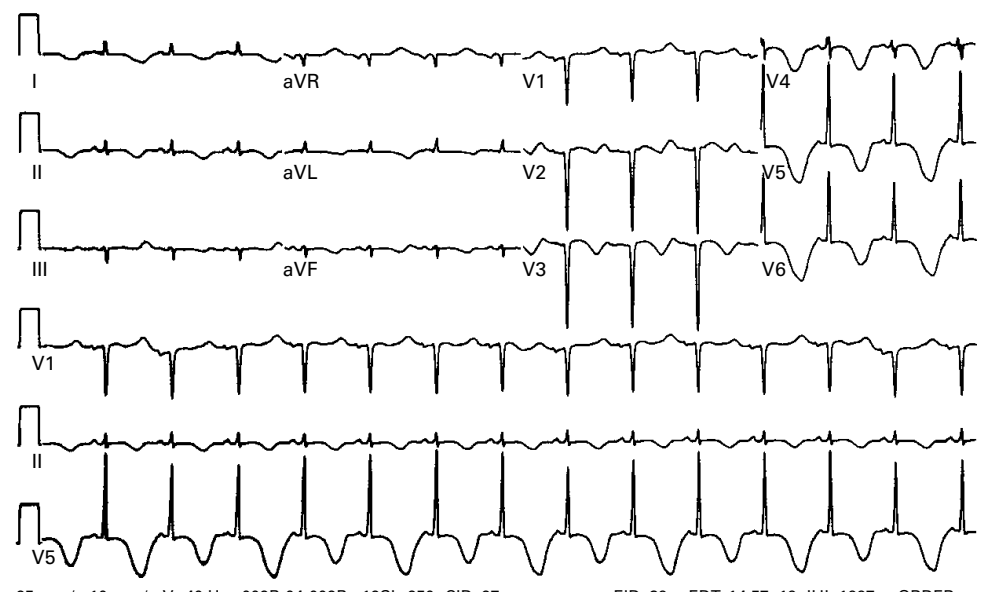

suddenly collapsed and had a cardiac arrest. She had ventricular fibrillation, which successfully converted to sinus rhythm with defibrillation. An echocardiogram showed left ventricular hypertrophy with no other significant abnormalities. Her electrolyte profile was normal.

$\mathrm{T}$ wave alternans (or repolarisation alternans) is a rare electrical phenomenon, seldom observed on electrocardiography, and is defined as beat to beat variation in the polarity or amplitude of T waves signifying an inhomogeneity in the refractoriness of the myocardium, setting the stage for re-entry and facilitating onset of malignant ventricular arrhythmias.

In our patient, the massive stroke with mass effect (which is a well known cause of long QT), together with profound catecholamine release from such an event, probably set the stage for profound myocardial stimulation. Subsequently dispersion of refractoriness (compounded by left ventricular hypertrophy) may have created myocardial instability and precipitated a ventricular arrhythmic event leading to cardiac arrest. The presence of $\mathrm{T}$ wave alternans in the immediate pre-arrest setting was an important clue to the electrical instability of the myocardium, before ventricular fibrillation.

KARTHIK ANANTHASUBRAMANIAM VANJI KARTHIKEYAN vanji@pol.net 